\title{
The Value to the Modern Library of a Technical Services Department
}

Mr. Colburn is chief of technical processes, Northwestern University.

$\mathrm{T}_{\mathrm{t}}$ HE ORGANIZATION of departments of technical services in libraries to cover the processes of ordering, cataloging and preparing material for use is a comparatively new development, having taken place largely within the past Io years. Even in this comparatively short time, however, considerable discussion has arisen concerning the advisability of establishing such units, the purposes they are intended to serve, and the benefits to be derived from them. The articles by Raynard Swank ${ }^{1}$ and Alex Ladenson, ${ }^{2}$ and the papers presented at the A.L.A. conference in June $1948,{ }^{3}$ are typical of such discussions.

The purpose of this paper is not to defend such units, but to indicate some of the objectives sought in their organization and particularly to describe some of the means of attaining these objectives.

\section{Objectives}

Mr. Ladenson, in the paper referred to above, has stated very well the aims he has constantly kept in mind in organizing the work of his department at the Chicago Public Library. Supplementing the objectives which he sets forth, we may consider the reasons which have often been

${ }^{1}$ Swank, Raynard C., "The Catalog Department in the Library Organization," Library Quarterly i 8:24-

32, January 1948. "The Acquisition and Preparation Departments," Library Quarterly 18:200-0 5, July 1948 . 3 "The Technical Services Division in Libraries: A Symposium," College and Research Libraries 1 0:46-68, January 1949. advanced for the creation of technical service departments.

One of these is decreasing the span of control of the librarian. There are many drawbacks to having a large number of lines of communication between the librarian and the various departments of the library. If regularly scheduled meetings of department heads are held, it is rather difficult to transact business or discuss and establish policies if the membership of the council is too large. That is an aspect which makes a large committee practically as useless as no committee at all. If the communication is by means of individual conferences, a large part of the librarian's time is consumed by these conferences, to the detriment of his other duties. In either case, situations are bound to arise in which one division head will raise a problem which involves another closely related division. Let us say that the head of the order division reports the imminent receipt of a large collection of material. Such an event inevitably requires special planning by the head of the cataloging unit and the person in charge of mechanical processing to avoid the creation of a bottleneck. A consultation with the librarian of the three people involved is then indicated. Perhaps ideally it might at times be possible to do this without the librarian's participation, but in practice the tendency to view the problem only from the point of view of one's own division makes this inadvisable. If, however, there is one person responsible for the work of all three units, he can conduct 
the necessary planning, referring to the librarian only those matters which involve general library policy.

Another objective sought in the creation of a technical services department is an increase in the flow of materials. There is probably no library of any size which does not have somewhere within it a backlog of some magnitude of materials awaiting processing. I do not mean to imply that such a backlog can be eliminated overnight by the creation of a technical services department, but certain steps can be taken and some results achieved through this medium. Of these I shall have more to say later.

Cooperation among divisions within the department is another objective of the technical services department. Everyone of us has a tendency, more pronounced in some cases than in others, to look at matters from the point of view of our own section or part of the work. This is natural and inevitable, and will continue to be as long as human beings and not robots are doing library work. The appointment of one person to supervise the various aspects of processing makes it possible to have an individual whose prime allegiance is not to any one unit. He or she will thus have a broader point of view and can plan and direct the work with more objectivity. This person can also tactfully indicate to the various division heads the problems of the others and create a better understanding of their mutual difficulties.

Costs of processing have long been a matter of concern to librarians, and also to boards of trustees. I know of one member of the board of a large library who became convinced that the processing costs were much too high. Accordingly, he constituted himself a committee of one to investigate the matter, much to the embarrassment of the librarian, and even more to the head of the catalog department. This trustee simply could not be convinced that after a book was purchased, it was necessary to allow an amount almost approaching the purchase price to prepare it adequately for use by the library's patrons. We are all only too well aware that books do not arrive in the library completely ready to be placed on the shelves or issued by the circulation desk, and that cards for them do not miraculously appear in the catalog. I do not pretend to know what is the proper percentage of the library budget for processing, or what the average cost for processing a book for use should be. Statistics of this type are difficult to obtain, and figures for the average cost of processing a book are unreliable at best, as they do not take into account other work done by the processing units.

But we must admit that the processing administrator and the librarian will do well to consider the possibility of reducing costs, especially in these times when gifts of money are decreasing in size, and requests for appropriations are being more carefully scanned by those in charge of allotting funds. The technical processes organization may be able to help in the reduction of such costs.

\section{Correlated Order Forms}

One device which has attracted wide attention since its appearance a little more than 5 years ago is the correlated or multiple order form. By this term is meant any or all of the various systems of preprinted order forms which come in a varying number of copies up to nine and perhaps more. Most of these are precarboned, although some are not. Those which are generally incorporate the snap-out principle for the removal of the carbons after the typing operation is completed. They may come in pads containing one to five sets of forms, or in long strips composed of several hundred sets. I should like to review the procedures followed with ours at Northwestern University to indicate how we are attempt- 
ing to streamline our work by their use. We do not claim that our forms as now constituted are perfect. In fact, we are already planning changes to be made when the time comes to reprint them. Nor do we maintain that they are superior to those in use at other libraries. The sole reason for using them as examples is my familiarity with them.

The forms consist of seven parts, all bearing the same serial number in red, and come in strips of 400 . When the typing operation is completed, the sets are detached from the strip, the carbon paper snapped out and discarded. The individual parts can then be disposed of, as will be shown later. So much for the mechanical details.

Requests for material may be received on form slips provided for the purpose, or simply in the catalog or list received from the dealer. In either case the searching is done by use of the medium supplied, that is from the slip, catalog, or list. In the searching process, any bibliographical information which may be of use in ordering the book, or in aiding the dealer in identification of the item, is added to the citation. If this is of help to the catalogers also, so much the better. The items are then sorted according to the dealer with whom they are to be placed, if this is not obvious from the source of the quotation, and given to the typist, who prepares the order as indicated above.

Part one is the "original purchase order." Information typed upon this includes, in addition to the author, title, place, publisher, date, and other necessary bibliographical data, the fund to which it is to be charged, the name of the dealer, the date of order, the number of volumes if more than one, the number of copies desired, the eventual location of the book (if other than the main stacks), the person and department making the recommenda- tion, the source of the quotation, and the estimated or list price. This information, by means of the carbon, carries through to all the other parts of the set. The lower portion, which differs on each part, gives general directions to the dealer for filling the order. These are supplemented by more detailed instructions on the verso of the slip. Experience has taught us that too lengthy instructions are of little value, as the dealer will not take the time necessary to read them. This part goes to the dealer and he can keep it for his records if he so desires.

Part two is a report slip which also goes to the dealer. Prominently printed in red upon this slip is the instruction "Return this slip with the book." By having this slip in the book when it arrives, we are able to locate those other parts of the form which are filed by order number, as well as those which are filed alphabetically. It is also possible to shelve the books numerically by order number while they are awaiting checking in or other steps in the processing. At the bottom of this second part is a note to the dealer requesting him to return the slip with the book if it is available, or if not, to report on the reverse side where he may check in the spaces provided any of several listed reasons for nondelivery. This saves correspondence on the part of the dealer. After the book has been checked in and forwarded for processing, this report slip is used as the permanent record in the "dead file." Here it will in the future answer questions concerning source, date of purchase, cost, etc., in case of loss or necessity of replacement.

Part three is the fund slip which is used in the bookkeeping procedure, of which more will be said later.

Part four is the order slip for Library of Congress cards. The bottom of this slip contains the report symbols to be used by L. C. when cards are not available. These 
slips are forwarded to the Catalog Maintenance Section, which is responsible for card ordering. Cards for current imprints are ordered immediately. For noncurrent items, the order is placed when the material is received. If the card number is found in the verification or searching process, it is supplied on the order forms at the time of typing. If not, cards are ordered by author and title. The preparation of the card orders formerly occupied the major portion of one assistant's time. Now much of it is done in the same process as the typing of the purchase order.

Part five is a claim slip. This is used as a follow-up in cases where the book or a report is not received in a reasonable length of time. The length of time an order is outstanding can easily be determined by checking the fund file, which is filed by order number, and is therefore a chronological record. A note to the dealer at the bottom of the slip states that no answer has been received on the order, and requests him to supply the book or report in the spaces provided on the reverse side of the slip. These are identical with those on the report slip. Use of these slips saves writing letters to trace items not received.

Part seven is the temporary catalog card. This is filed in the public catalog immediately, before the order is mailed. Thus we have an additional check to avoid duplication. The bottom of the slip bears a note to the user of the catalog, informing him that the title is on order or in process. If the item is urgently needed it can be requested at the circulation desk. If it has been received, the cataloging can be speeded or the book used in the library at once. By placing these cards in the public catalog, we have only one place to search before ordering. We also have a record of the presence of the book from the time of its receipt, a fact which is much appreciated by the faculty.
The original request slip by which the order was originated by faculty or staff member has meanwhile gone with the book through the cataloging process. In this way it conveys to the cataloger any bibliographical data obtained in the searching process. Finally, it is used to notify the person originally making the request that the book is now ready for circulation.

By the use of these slips, we have reduced the number of typing operations, the number of places in which searching must be done before ordering, and the amount of time required in preparing $\mathrm{L}$. C. card orders. We have also devised a means of conveying available information to the catalogers, and of providing temporary cataloging for our purchases. There are undoubtedly other uses which can be made of these forms. We are now considering the possibility of using one part as a binding slip, but this is still in a very tentative stage.

\section{Bookképing}

On the basis of the multiple order slip system I have just described, we have also devised a new accounting and bookkeeping system for book purchasing. The amount of bookkeeping necessary varies in different libraries, according to the regulations of the institution or governmental system of which the library may be a part. Therefore, a good system for us at Northwestern may not be satisfactory for another library, but I should like to explain briefly what we have worked out, as an indication of the possibilities for the streamlining of this procedure in a department of technical service.

As has already been indicated, our bookkeeping system depends very closely upon our multiple order forms. One slip in the set is called the "fund slip," and is designed primarily for accounting use, although it also serves other purposes. By agreement with the business office of the university, bills are sent through for pay- 
ment three times a month. Foreign bills go to the business office on the third of the month, in order to allow time for processing and payment by the 3oth for inclusion on the monthly statement of budget expenditures. Domestic bills go through on the 12th and 22nd. These dates were chosen to avoid conflict with bills coming from other parts of the university. This cooperation between the business office and the library is indicative of what can be done by the head of a technical services department. Perhaps it could be done as easily by the order librarian, but in our organization at least, it had not been done previously. As the payment periods are thus spaced at intervals of ro days, we have adopted these periods as basic in our scheme. In other words, the books are completely balanced, showing commitments, expenditures, cash balance and free balance at these intervals.

Each day as orders are prepared, the fund slips are forwarded to the bookkeeper. They are divided by fund, and are placed in a separate part of the file until the time for balancing the ledger arrives. At that time the commitments, i.e., the estimated or list prices for items ordered on each fund, are totaled and entered in the column of the ledger provided for commitments outstanding, and the estimated free balance reduced accordingly.

The dealers are requested to indicate on the bills rendered the name of the fund and the order number for each item. This simplifies the pulling of the fund slips as the bills are processed. When the bills are ready for payment, the fund slips for items received on each fund are collected and the totals of commitments and actual expenditures computed. The commitments thus paid are entered in the ledger column for this purpose, and the balance of commitments outstanding reduced accordingly. The total of expenditures for the period are also entered in the ledger, the total to date computed and the cash balance reduced accordingly. Since the estimated price is only an approximation and does not take into account the discounts which will be given by the dealer, it is also necessary to adjust the estimated free balance. This is easily done by computing the difference between estimates and expenditures. If we have overestimated, this difference is added to the free balance. In case we have underestimated, the difference is subtracted.

Once each month a report of the status of the individual book funds is prepared for the librarian on a form designed for the purpose. He is thus in a position to see the manner in which funds are being expended and which departments are spending too rapidly and which too slowly. As the end of the fiscal year approaches, it may be necessary to prepare the report more frequently. Meanwhile, it is possible to determine from a glance at the ledger the status of any fund up to a point not more than Io days previous. Or we can prepare a report on the status of all funds in the time it takes to type out the form, approximately an hour. The preparation of the report formerly required two days or more, as it was necessary to recompute the outstanding commitments each time a report was made.

Thus far this system has worked very satisfactorily for the library and the business office. One request for information on the bookkeeping procedure has been received, with the announced intention of considering it for adoption in another large university.

The fund slips also serve other purposes. Since they are filed by order number, and the numbers are chronological, it is very easy to see what orders have remained unfilled for a long period. As the slips are transferred to another file after payment, they serve as a record of the purchases on each fund during the fiscal year, if such in- 
formation is desired. We have also kept the slips of items reported as unavailable by the dealer. These may give us some insight into the types of material which we are failing to obtain.

\section{Consolidation of Files}

In some libraries there is a duplication of files between the order division and the cataloging or other divisions. This situation inevitably leads to extra work, some of which can be avoided by consolidation and cooperation. In a number of institutions where correlated order forms are not used, an order file is kept as well as an inprocess file of material received and awaiting processing or being processed. These two files can often be united. The order slips can then be marked to show receipt of the volume and immediately replaced in the file, thus saving one filing operation. Frequently double records are kept for periodicals, the one in the order division showing, in addition to the status of the library's holdings, decisions upon continuing subscriptions and filling gaps, while the one in the periodical division gives the holdings and the binding status. Assuming that the divisions are sufficiently close together in location, one file can often be utilized to contain all this information.

\section{Personnel}

The head of the technical services department is often in a better position than the division head to separate the clerical from the professional duties of the staff. The greater objectivity which can be achieved in this position, and the over-all view afforded, contribute to this. Moreover, greater flexibility is possible in shifting clerical personnel from one division to another as occasion demands, or in the sharing of the time of such personnel by two divisions. Nor is such flexibility confined to the clerical staff. Professional workers can also be shifted if their qualifications permit and the interests of the department so dictate. Since the head of the department is aware of special problems or an increased work load in one division, plans can be formulated to avoid breakdowns in processes in all divisions of the department by advance preparation or temporary re-alignments. The flow of work can be maintained more evenly and the transmission of information expedited.

As a result of this same over-all viewpoint, the head of the technical services unit is in a better position to draw up realistic job specifications and to evaluate the relative importance of the various positions in the department. Thus a more equitable salary scale can be achieved, and a more reasonable budget recommendation prepared for presentation to the librarian. Individual staff members would thus seem to be assured of a fairer evaluation of their and their position's worth in relation to the other people and positions in the department.

The centralization of the administrative organization for the order, cataloging and mechanical processing divisions means that one person is concerned with personnel records, such as absences, vacations, and leaves. It seems reasonable that one person can keep such records for 60 people more quickly and easily than can three persons for 20 people each. Moreover, centralization of such records provides for greater uniformity in the application of rules pertaining to such matters.

\section{Relations with the Public}

Relations of a library with the public is a matter which should be of prime concern to the staff. From the point of view of technical services, we have on the one hand the dealer. Whatever we may say concerning individual agents, we must agree that the majority of them are willing and even 
anxious to cooperate with their customers, the libraries. The head of a technical services department is in a good position to smooth the path toward better relations in this sphere. If he knows his work, he is conversant with the problems of the order division, the catalogers, the serial records staff and the people preparing material for binding. With this knowledge, he is better prepared to present the over-all problems of the library to the dealer and to work out means of cooperation with him. On the other hand there are the faculty and the student body. In his relations with them the technical services specialist is able to bring about better understanding by developing ways and means of getting the ma- terials they need into the library more. quickly, and of cataloging and classifying them more satisfactorily. The resulting satisfaction will pay large dividends.

These are only some of the numerous devices which can be utilized in a technical services department to streamline and improve operations. More can be found, and in the discovery and application of these the specialist in this field will find a constant challenge and a rewarding satisfaction. This is not a static field, but a constantly changing one, which offers great possibilities to the adventurous. Life is never dull, and the person entering this field can expect to enjoy his work while helping to improve library technique.

\section{Agricultural Research and the Exchange Problem}

\section{(Continued from page 46)}

Directory of Agricultural Libraries and of Libraries Specialized in Subjects Related to Agriculture (Rome, The Institute, 1939. 3 I Ip.) Revisions of other lists issued by the International Institute of Agriculture, such as the International Directory of Animal Husbandry Institutions (Rome, 1933. 322p.), the International Directory of $\mathrm{Ag}$ ricultural Engineering Institutions (Rome, 1939. I 52p.), the International Directory of Dairying Institutions (Rome, I934. 450p.), the International Directory of $\mathrm{Ag}$ ricultural Experimental Institutions in $\mathrm{Hot}$ Countries (Rome, 1934. 563p.), and In- stitutions Agricoles dan les Pays Tempérés (Rome, 1933. 306p.), would also be of great value. We could certainly attack, through regional committees, the question of which libraries were to be designated as depositories for duplicates of foreign agricultural material received by the larger research libraries. In any case I propose that we attempt to do something concrete about the foreign exchange situation. If enough interest is aroused I see no reason .why a decision in favor of decisive action cannot be reached at the national meeting of the A.L.A. in July 1950. 